ACTA UNIVERSITATIS LODZIENSIS

Folia Litteraria Romanica 16, 2021

https://doi.org/10.18778/1505-9065.16.14

Florian Koch

Université de Bourgogne

(iD) https://orcid.org/0000-0002-4897-7174

florian.koch@u-bourgogne.fr

Marie-Anne Berron

Université de Trèves

(iD) https://orcid.org/0000-0002-7624-6686

berron@uni-trier.de

\title{
La formation d'une identité collective à travers les expressions de l'amour et de la haine : une analyse exploratrice des discours de supporters de foot en Allemagne et en France ${ }^{1}$
}

\begin{abstract}
RÉSUMÉ
Le foot représente de nos jours un exemple type de plateforme par laquelle les dynamiques sociales des sociétés peuvent être observées. Les forums sont des lieux virtuels riches en expressions quotidiennes où les supporters de foot expriment leur « amour » ainsi que leur « dédain » vis-à-vis de l'autre. Nous effectuerons une analyse de contenu au cours de deux saisons de l'Olympique de Marseille et du Bayern de Munich ainsi que du Toulouse FC et de Hanovre 96. Cette analyse illustre que les expressions haineuses qui démarquent les groupes sont déterminantes pour la formation d'une identité collective. De plus, l'analyse confirme que la croyance collective correspond aux performances des équipes. Tandis que les top-clubs se définissent par leur performance sportive de manière positive, les clubs relégables se définissent plutôt de manière négative - ressentant une forme d'infériorité - afin de se démarquer, ceci tout en restant fidèles à leur propre club comme le démontre leur devise.
\end{abstract}

MOTS-CLÉS - identité collective, foot, expression de la haine, expression de l'amour, Allemagne, France

${ }^{1}$ Cet article se base sur les conceptions du projet de recherche EUFoot. Nous remercions donc Alexander Brand et Regina Weber de l'Université Rhin-Waal ainsi qu'Arne Niemann de l'Université Johannes Gutenberg de Mayence. 
The Formation of a Collective Identity Through Expressions of 'Love' and 'Hate': An Exploratory Analysis of the Discourse of Football Fans in Germany and France

\begin{abstract}
SUMMARY
Football is an exemplary platform where the social dynamics within and between societies can be regularly observed. In addition, online message boards are a rich resource of everyday expressions where football fans express their 'love' of their clubs as well as their 'disdain' for others. We conducted a qualitative content analysis during two seasons of Olympique de Marseille and FC Bayern Munich as well as Toulouse FC and Hannover 96. Our analysis illustrates that hateful expressions that differentiate groups are much more prevalent and therefore more determinant in the formation of a collective identity. Furthermore, the analysis confirms that the self-conception corresponds to the performance of the teams. While fans of top clubs portray themselves in a positive way because of their team's sporting performance, fans of teams who are battling against relegation define themselves more in a negative way - feeling a form of inferiority - in order to differentiate themselves whilst still remaining attached to their own club as the club motto implies.
\end{abstract}

KEYWORDS - collective identity, football, hateful expression, expressions of affiliation, Germany, France

\title{
Introduction
}

« Un seul sport n'a connu ni arrêts ni reculs : le football. A quoi cela peut-il tenir sinon à la valeur intrinsèque du jeu lui-même, aux émotions qu'il procure, à l'intérêt qu'il présente ? " (Pierre de Coubertin, 1897). Cette citation montre bien que le sport et plus spécifiquement le football jouait déjà un rôle essentiel dans la société et que Coubertin en avait déjà prédit l'importance à venir. De nos jours, le football prend une ampleur considérable et il est fréquent de voir qu'il déclenche des passions irrationnelles de la part des fans. Les émotions y jouent un rôle primordial et peuvent aller dans des dimensions diamétralement opposées telles que la haine (exclusion du groupe ou rivalité entre groupes), ou l'amour (inclusion dans le groupe). Qui dit 'groupe' sous-entend également identité. Chaque groupe possède donc une identité (croyance collective et conception de soi) dans laquelle il exprime des émotions liées à l'amour et à la haine.

Le football reflète bien-sûr (partiellement) les sociétés modernes en créant à la fois une cohésion forte à l'intérieur des groupes formés - éventuellement seulement temporaire pour un évènement spécifique - mais également des clivages ou bien des conflits latents entre les groupes pouvant être arbitrairement formés. Le foot permet plus particulièrement d'observer avec clarté le concept d'identité multiple superposée comme l'interaction des identités nationales, régionales et urbaines (King, $2000: 427$; Brand, Niemann, 2014 ; 44 et suivantes ; Brigevich, $2018: 642$ et suivantes). La structure bipolaire simple du foot avec ses deux équipes - ses deux groupes de supporters - et normalement seulement un vainqueur - offre une plateforme idéale pour explorer les expressions de l'amour et de la haine qui sont fortement reliées l'une à l'autre (Winands, 2015 : 204). 
Nous assumons que le foot est un vecteur important pour la formation d'une identité collective - en particulier parmi les groupes de supporters dans le foot professionnel. Cet article veut donc, dans un premier temps, évoquer le cadre théorique adopté en présentant la triangulation du concept d'identité ainsi que les trois dimensions de l'identité collective (2). Le tout avec l'objectif de déterminer cette même notion d'identité collective dans le domaine du football et à l'aide de citations tirées de forums de supporters de foot. Dans un deuxième temps, nous poserons les questions de recherche auxquelles nous souhaitons répondre (3) et le point sera fait sur la méthodologie utilisée (appliquée) (4). Nous nous baserons ensuite sur les résultats concrets d'analyse de forums de supporters (5) pour répondre en conclusion (6) aux questions posées.

\section{Cadre théorique}

Il faut donc partir de la notion d'identité pour comprendre l'identité collective des supporters de football. Selon Brubacker and Cooper (2000:6) le terme de l'identité est à utiliser plutôt dans le cadre d'un concept analytique « hopelessly - ambiguous ». Ils proposent ainsi soit de refuser complètement ce concept d'identité, soit de développer des méso-conceptions, donc des modèles, qui concrétisent le terme d'identité. À cette suite, nous ne proposons donc pas ici une définition de l'identité mais un modèle cherchant à mesurer des aspects identitaires spécifiques. Ainsi le cadre théorique de cet article utilise la notion d'identité soumise à une triangulation théorique (1.1) dans laquelle la notion d'identité collective contient elle-même trois dimensions différentes (1.2).

\subsection{La triangulation théorique du concept de l'identité collective}

Pour comprendre le concept d'identité collective des joueurs de foot, nous appliquons une triangulation théorique selon Denzin (1989: 236 et suivantes) en combinant trois concepts issus du terme de l'identité et fortement interdépendants, à savoir l'identité sociale (1), l'identité collective (2) et la théorie du prototype (3).

Selon la théorie de l'identité sociale, tous les individus convoitent une identité sociale positive (Tajfel, Turner, $2004: 282$; Weber, $2018: 70$ et suivantes). Celleci se produit par les affiliations d'un individu à un groupe. Le sentiment positif d'appartenance à son propre groupe - autrement dit à son 'ingroup' - a un effet d'exclusion par rapport aux groupes « autres » concernés - dénommés 'outgroup'. $\mathrm{Si}$, toutefois, son groupe respectif est jugé trop négatif, les individus appliquent des stratégies spécifiques pour améliorer à nouveau leur identité sociale. Outre le simple changement de groupe ou le ré-étiquetage d'attributs prétendument plus positifs, l'autre groupe est souvent dévalué, parfois massivement, afin de valoriser son propre groupe (Geschke, $2012: 36$ et suivante). 
L'identité collective souligne qu'une identité est partagée par un collectif, donc qu'elle est impensable sans d'autres personnes impliquées. De plus, l'approche accentue l'idée que l'identité du groupe - comprise comme trait caractéristique d'un groupe et représentant un aspect du « concept de soi » d'un groupe entier ou bien une sorte de « croyance collective »- est désormais complètement dissociée de l'identité individuelle et existe même si les personnes arrêtent de s'y identifier (Weber, $2018: 25$ et suivantes).

Par conséquent, l'identité collective conduit, au moins partiellement, à une dépersonnalisation car à la fois les attitudes et le comportement sont désormais considérés aussi bien par le 'ingroup' que par le 'outgroup' comme caractéristiques typiques d'un groupe spécifique. Selon Eleanor Rosch (1983: 73 et suivantes), ce " prototype » se rapproche le plus possible du stéréotype de son propre groupe pour créer une différenciation maximale par rapport aux groupes « autres ». L'identité individuelle est absorbée dans le prototype de groupe. Ce qui implique donc un oubli de sa propre identité au profit de l'identité du groupe en place. En conséquence, Wenzel et al. (2007 : 335) définissent le prototype comme « ideal-type member of a category, that best represents its identity in a given context and frame of reference ».

\subsection{Les dimensions de l'identité collective}

La notion d'identité collective est alors exploitée en créant trois dimensions (voir Figure 1 ci-dessous) - celle de l'autodéfinition (concept de soi-même), celle du groupe d'appartenance et celle du cadre de référence. Ces trois dimensions permettent de comprendre les détails de l'identité collective des supporters de foot.
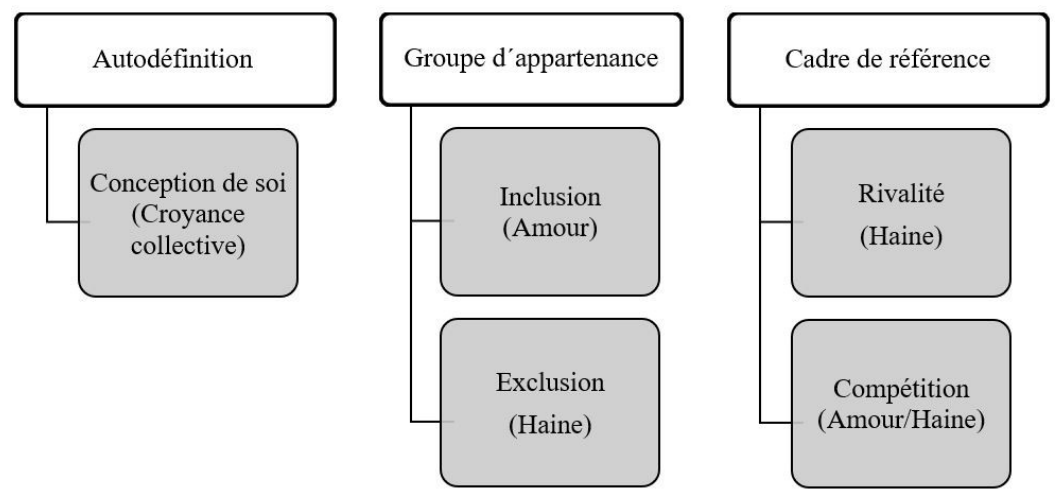

Figure 1. Les trois dimensions de l'identité collective²

\footnotetext{
${ }^{2}$ La Fig. 1 reprend les réflexions de Weber et al. (2020: 299 et suivantes) et y rajoute la catégorie de l'autodéfinition.
} 
L'aspect dimensionnel de l'autodéfinition est composé principalement par le « concept de soi » appelé aussi « la croyance collective ». L'aspect dimensionnel du groupe d'appartenance contient deux sous-catégories majeures : l'inclusion et l'exclusion. La dimension du cadre de référence comprend les sous-catégories « rivalité » et « compétition ».

L'analyse du corpus dans la suite de cette exploration permettra d'identifier les expressions de l'amour et de la haine en les élargissant aux expressions de l'admiration, de l'affection et du mépris, voire du dénigrement, et en les reliant aux catégories créées précédemment.

\section{Questions de recherche}

En partant donc du cadre théorique de la triangulation (identité sociale, identité collective et théorie du prototype) et en se focalisant sur les 3 dimensions de l'identité collective et les sous-catégories, à savoir l'autodéfinition (concept de soi et croyance collective), le groupe d'appartenance (inclusion et exclusion) et le cadre de référence (rivalité et compétition), il est possible par l'analyse du corpus présenté dans la $2^{\text {ème }}$ partie de ce travail de mieux comprendre et d'effectuer une approche de l'identité collective des supporters de foot. Nous avons formulé sur cette trame les deux questions de recherche $(\mathrm{QR})$ suivantes :

QR1 : Quelles expressions, que ce soit celles de l'amour ou celles de la haine, sont les plus déterminantes pour la formation collective de l'identité ?

QR2 : Comment les « concepts de soi », appelés aussi « croyances collectives », sont-ils exprimés à travers les expressions de l'amour (appartenance) et de la haine (démarcation) dans les discours des supporters de foot professionnel ?

\section{Méthodologie et sélection de cas}

Pour essayer de répondre à ces questions nous procédons à une analyse exploratrice du contenu (Mayring, 2016:50 et suivantes) de forums de supporters officiels de deux équipes en Allemagne et de deux équipes en France. Nous avons tout d'abord généré un système par catégorie reprenant : la conception de soi, les expressions de la haine face à son propre club et au club adverse, face aux autorités comme la Fédération Française du Football (FFF), et les expressions de l'amour face à son propre club. Ce système fonctionne à la fois d'une manière déductive dans la mesure où nous avons repris le cadre théorique mais également d'une manière inductive car nous avons analysé les données contenues dans le corpus en les catégorisant.

Pour couvrir le mieux possible les deux pays, un top-club ayant une perspective européenne ainsi qu'un club relégable avec une ambition au niveau national ont été choisis dans chacune de ces ligues, donc deux clubs typiques par pays et dans 
un contexte spécifique afin de percevoir les différences de comportement des supporters selon la perspective nationale ou internationale du club.

Afin d'obtenir à la fois des représentants typiques et atypiques, deux saisons -2016/2017 et 2017/2018 - avec 35 discussions sur des forums contenant plus de 35.000 posts ont été analysées.

\section{Cadre empirique et analyse des données}

La partie qui suit veut donc présenter une analyse concrète de forums de supporters en France et en Allemagne de clubs candidats au titre de champion, le Bayern de Munich et l'Olympique de Marseille, et de clubs relégables : Hanovre 96 et le Toulouse FC. Pour ce faire, les expressions liées aux trois dimensions de l'identité collective ainsi que leurs sous-catégories - concept de soi et croyance collective, inclusion (amour) et exclusion (haine), rivalité et compétition (haine) - seront reprises et analysées pour définir dans quel contexte elles apparaissent le plus fréquemment. Ne seront présentés ici, de façon qualitative, que les expressions les plus marquantes du corpus pour illustrer les résultats de l'analyse exploratrice du contenu.

Nous constaterons donc, dans l'analyse de forums, que les supporters adhèrent par leur devise à une conception de soi voire à une croyance collective. Dans ce cadre, ils ont alors un groupe d'appartenance au sein duquel ils vont avoir recours à des expressions verbales symbolisant l'inclusion (amour) et l'exclusion (haine). Ces expressions d'inclusion et d'exclusion seront utilisées si la réussite du club concerné correspond aux attentes ou non de leur cadre de référence lors de compétitions. Ces compétitions génèrent d'ailleurs bien souvent des rivalités s'exprimant par l'usage d'expressions de haine voire d'amour.

\subsection{Clubs : candidats au titre de champion}

Les clubs choisis, le Bayern de Munich et l'Olympique de Marseille, sont des clubs ayant une ambition européenne. Ils sont représentatifs de l'Allemagne et de la France et font partie des top-clubs.

\subsubsection{Le Bayern de Munich}

L'analyse des données montre que les supporters du Bayern de Munich définissent leur club comme un top-club au niveau européen avec une longue tradition et une très grande base de supporters internationalisés.

Il faut souligner l'importance de la cohésion sociale et la confiance des supporters envers leur club exprimée déjà par le nom de « Bayern » et surtout par la devise 
formulée en dialecte régional « nous sommes nous » (« mia san mia »), confiance que montre aussi l'expression suivante tirée du corpus ; « cependant avec beaucoup de 'mia san mia' et un peu de l'odeur de notre écurie, ça marchera ».

Le simple fait que la devise du club soit si usitée montre que la conscience collective du groupe est rattachée à l'image de soi du supporter.

Leur attachement passe majoritairement par l'attaque verbale envers le groupe rival, donc par l'expression de la haine (l'exclusion). Les supporters démarquent donc leur groupe tout particulièrement à travers les expressions de haine envers les autres surtout au niveau européen comme le montrent les exemples du tableau suivant : «Cette fois, le coq [Cristiano Ronaldo] sera plumé correctement. » ou encore « Oh, le 'yeux globuleux' [Mesut Özil] a le droit de jouer. » Ces deux exemples montrent clairement l'attaque vis-à-vis des joueurs adverses afin de les dévaloriser. Le Real Madrid, le grand rival au niveau européen et Cristiano Ronaldo comme joueur vedette sont dans le collimateur des supporters, comme le montre la citation suivante ${ }^{3}$ avec sa féminisation du nom propre de Cristiano Ronaldo : « La princesse Cristiana Ronalda fait de nouveau la tronche... ».

On observe donc des citations de haine envers le club adverse mais également envers son propre club lorsque les résultats ne sont pas à la hauteur des espérances des fans comme dans l'exemple ci-dessous : «Toute l'Europe est sur le pied de guerre et nous nous détruisons nous-même. ». On observera cependant que les remarques liées à leur club sont largement moins dénigrantes que celles envers le club adverse. Elles sont plutôt liées au niveau des joueurs et à la peur de perdre plutôt qu'à une mauvaise préparation. Malgré la prédominance des expressions haineuses, les supporters recourent tout de même à des expressions d'amour donc d'inclusion et de croyance collective : " Quand on lui fait sa place, ce Franck [Ribéry], il a encore et toujours une classe mondiale ! ...» ou encore « Je le kiffe de plus en plus notre gitan ».

\subsubsection{L'Olympique de Marseille}

Les supporters de l'Olympique de Marseille définissent leur club comme un ancien top-club au niveau international avec une grande tradition liée à de nombreux succès - comme le montre leur devise officielle : «Droit au but » marquant l'objectif d'être vainqueur - et une grande base de supporters fanatiques. En outre, ils se définissent comme le leader du championnat des « clubs purs ».

Cependant, bien que le corpus contienne des expressions liées à l'amour des supporters envers leur propre club, elles sont peu nombreuses ( Ce maillot, c'est un honneur. Je viendrais même pour porter l'eau ») tandis qu'il y a énormément

\footnotetext{
${ }^{3}$ Il est évident que féminiser un mot n'est pas négatif, mais c'est la vision misogyne qui en fait une insulte, et qui est associée au terme de princesse : c'est-à-dire un personnage en représentation, qui ne fait finalement pas grand-chose et est capricieux.
} 
d'expressions de haine envers leur propre club, surtout contre les joueurs et entraîneurs mais également envers les groupes de supporters comme l'illustrent les citations suivantes: "Thauvin qu'il commence par dégonfler son ballon de baudruche qui lui sert de cerveau ! » ou encore "Putain ... ces connards qui vont au stade et qui ne font les déplacements que pour se battre ... Je n'ai aucune espèce de respect pour ces animaux ... ». Florian Thauvin est un joueur du club et en parlant de ces " connards » l'internaute se réfère à certains supporters de l'Olympique de Marseille. Il y a donc un idéal des résultats à avoir par le club qui ne semble jamais être réalisé, ce qui suscite une vive critique des supporters. Les arbitres sont très souvent déclarés responsables de la défaite du club et se font donc vivement critiquer : " mais quel enculé cet arbitre !!!! Il vient d'où ce charlot ??? Les mecs... » ou encore « Ce championnat c'est de la merde en boite avec des arbitres pourri $»$.

Les supporters semblent également, d'après certaines citations, se diviser en deux groupes distincts : d'un côté ceux qui se considèrent comme des supporters et d'un autre côté les 'hooligans', sombrant rapidement dans la violence et n'ayant pas le respect du sport. Les supporters se sentent donc blessés dans leur identité et dévalorisent ainsi le groupe considéré comme adverse. Les principaux rivaux sont avant tout au niveau national, mais abordés dans un contexte européen. On repère donc l'exclusion des joueurs rivaux comme le montre la citation suivante : " Il est mignon ce faux derche [Franck Ribéry, ancien joueur de l'OM] ! : lol » ou des remarques d'exclusion, donc de haine, liées à la rivalité entre clubs : « Les prétentieux Parisiens, les donneurs de leçons Lyonnais ainsi que ces couillons de journaleux bien pensants (...) vont pouvoir regarder les matchs de coupe d'Europe (...) sur leur canapé kleenex à main !».

\subsection{Clubs relégables}

Les clubs relégables choisis, Hanovre 96 et le Toulouse FC, sont représentatifs de clubs jouant à un niveau national sans ambition internationale. Leurs supporters respectifs ont une identité régionale et des rivalités intra-nationales fortement marquées.

\subsubsection{Hanovre 96}

Les supporters de Hanovre 96 définissent traditionnellement leur club comme relégable : " Il est de nouveau plaisant de regarder jouer H96 »- et ayant une bonne base de supporters engagés, comme le montre leur devise « jamais seuls ». Cependant, les supporters se sentent inférieurs par rapport à d'autres clubs, comme le prouve la citation suivante : « 96 est probablement aussi attrayant et intéressant pour les joueurs qu'une femme variolée de 80 ans pour un adolescent ». Ils utilisent 
souvent des propos haineux envers eux-mêmes (club ou supporters) comme le montrent les citations suivantes : « Le voyage repart juste à nouveau en direction de ce club de merde !» ou encore « Pour un supporter trop peu d'événement sportif, pour un client trop peu de sensation. Mais les saucisses et la bière chez les élites étaient délicieuses ». Cette dernière citation les dévalorise en tant que club mais valorise en parallèle un club concurrent. Le rival principal est le club voisin de Brunswick.

Cependant, bien que de nombreuses expressions liées à l'exclusion et à la rivalité face à des clubs adverses (donc à la haine) soient présentes dans le corpus de ce club, celles-ci ne franchissent pas un certain registre de langue, ne tombant ainsi pas systématiquement dans le vulgaire : "Pour la pompe à air [Antonio] Rüdiger, Chelsea a déposé 38 millions d'euros » ou encore «Espérons qu'ils se feront botter les fesses par le Real, les salauds ». Les expressions liées à l'inclusion donc à l'amour envers leur propre club sont rares : " Mais j'irais aussi dans le championnat régional avec 96 pour revenir avec le phénix rouge, si seulement ces grands maîtres étaient enfin partis » ou encore «'Fülle' [Niclas Füllkrug] est un peu 'on fire'» et certaines le sont même envers les clubs adverses : « Le BVB fait simplement un excellent travail ».

\subsubsection{Le Toulouse FC}

Les supporters du Toulouse FC définissent traditionnellement leur club comme club relégable mais ayant également une très faible base de supporters capables de souffrir pour leur région et leur ville selon leur devise « La Garonne est viola » qui marque très fortement leur identité : «Et un club, ce sont d'abord, des valeurs, une identité. Je dirais même... ». Certaines citations du corpus mettent en évidence un régionalisme et un attachement au club par devoir : "Qu'est-ce que j'ai fait au bon Dieu dans mes vies antérieures pour supporter un club pareil... Looser ad vitam éternam », "Ces équipes ne gagnent pas grand chose (...), mais je suis toulousain. Pas marseillais ni parigot. (...) je veux que les équipes de ma ville (...) se dépouillent pour le maillot ». Malgré tout, un attachement véritable est remarquable dans certaines citations marquant un lien fort envers leur propre club : « J'aurais donné un rein (mise en évidence originale) pour qu'on tienne ce 0-0 jusqu'à l'a 70'eme au moins. ».

Les expressions rassemblées sont souvent très dures, surtout les expressions haineuses, voire vulgaires. Les supporters sont vulgaires envers leurs propres joueurs comme le montre la citation suivante : "SORTEZ-VOUS LES DOIGTS DU CUL ! ET SERVEZ VOUS EN POUR VOUS SORTIR LES COUILLES DU SHORT BORDEL! » ou encore «Bon j'hésite à partir au stadium pour insulter les joueurs et PD malgré l'abo j'ai préféré regarder le match au chaud mais je crois que je vais profiter de la mi temps pour y aller et leur dire à quel point ils sont minables 
et à quel point j'ai honte d'eux », mais également envers les supporters du club : «C'est bien la preuve irréfragable de la déliquescence de l'Éducation nationale et de déclin logique de l'intellect gaulois. » ou envers les clubs adverses en tenant des propos racistes : «Inutile de se fatiguer à essayer de convaincre ce genre d'individus lorsque tant d'Oncle Tom pullulent avec des maillots de I'OM et du PSG. »

\section{Conclusion}

En guise de conclusion, on peut dire que cette analyse illustre bien que les expressions haineuses sont beaucoup plus répandues que les expressions de l'amour dans le discours des supporters de foot. On peut en déduire que, pour la formation d'une identité collective (QR1), les expressions haineuses - donc les expressions qui démarquent les groupes - sont plus importantes que les expressions d'amour qui - elles - sont inclusives. Il est à souligner que les expressions à la fois amoureuses et surtout haineuses sont majoritairement utilisées envers le groupe respectif.

En outre, les expressions de haine sont beaucoup plus répandues dans les forums français que dans les forums allemands. Qui plus est, les expressions haineuses semblent être plus utilisées dans le forum toulousain, donc un club français relégable. Les supporters de celui-ci expriment la haine différemment quand il s'agit du club adverse ou de leur club. Ainsi, envers leur propre club les remarques sont extrêmement dévalorisantes et vulgaires tandis qu'envers les clubs adverses elles sont souvent liées au racisme ou à des propos homophobes.

De plus, pour ce qui est de (QR2), l'analyse confirme la présomption selon laquelle le concept de soi, donc la croyance collective, correspond aux performances des équipes. Tandis que les top-clubs se définissent de manière positive par leur performance sportive, les clubs relégables se définissent plutôt de manière négative (ressentant une forme d'infériorité face aux clubs plus performants) afin de se démarquer mais ceci tout en restant attachés à leur propre club comme le démontre leur devise.

Finalement, les expressions haineuses sont majoritairement utilisées dans un contexte de rivalité. Les rivalités dépendent de la performance de l'équipe, même si les rivalités nationales voire régionales restent les plus intenses.

\section{Bibliographie}

BRAND, Alexander, NIEMANN, Arne (2014), "Football and National Identity in Europe », Panorama: Insights into Asian and European Affairs, vol. 1, p. 43-51

BRIGEVICH, Anna (2018), "Regional identity and support for integration : An EU-wide comparison of parochialists, inclusive regionalist, and pseudo-exclusivists ", European Union Politics, vol. 19, n 4, p. 639-662 
BRUBAKER, Rogers, COOPER, Frederick (2000), "Beyond "identity" ", Theory and Society, vol. $29, \mathrm{n}^{\circ} 1$, p. $1-47$

DE COUBERTIN, Pierre (1897), Souvenirs d'Amérique et de Grèce, Paris, Hachette

DENZIN, Norman K. (1989), The research act, Englewood Cliffs, NJ, Prentice Hall

GESCHKE, Daniel (2012), «Vorurteile, Differenzierung und Diskriminierung-sozialpsychologische Erklärungsansätze », APuZ, n ${ }^{\circ} 16-17$, p. 33-37

KING, Anthony (2000), « Football fandom and post-national identity in the New Europe », British Journal of Sociology, vol. 51, no 3, p. 419-442

MAYRING, Philipp (2016), Einführung in die qualitative Sozialforschung. Eine Anleitung zu qualitativem Denken, $6^{\text {ème }}$ édition révisée, Weinheim / Bâle, Beltz

ROSCH, Eleanor (1983) « Prototype classification and logical classification: The two systems » in New trends in conceptual representation (E. K. Scholnick éd.), Hillsdale, NJ, Erlbaum, p. $73-86$

TAJFEL, Henry, TURNER, John C. (2004), « The Social Identity Theory of Intergroup Behavior » in Political psychology : Key readings (J. T. Jost, J. Sidanius J. éds), New York, Psychology Press, p. 276-293

WEBER, Daniel (2018), Bedeutung und Wirkung gruppenbezogener Identifikation. Eine Analyse europäischer und nationaler Identifikation in Deutschland, Wiesbaden, Springer VS

WEBER, Regina, BRAND, Alexander, NIEMANN, Arne, KOCH, Florian (2020), " Non-elite conceptions of Europe : Europe as reference frame in English football fan discussions ?», Journal of Contemporary European Research, vol. 16, n 3, p. 293-319

WENZEL, Michael, MUMMENDEY, Amélie, WALDZUS, Sven (2007), « Superordinate identities and intergroup conflict : The ingroup projection model ", European Review of Social Psychology, vol. 18, no 1, p. 331-372

WINANDS, Martin (2015), Interaktionen von Fußballfans. Das Spiel am Rande des Spiels, Wiesbaden, Springer VS

Florian Koch - maître de conférences à l'Université de Bourgogne, enseignant à Sciences Po Paris. Diplômé d'une thèse de doctorat en cotutelle entre l'Université de Paris (Sorbonne) et l'Université de Leipzig, ses recherches portent sur les relations franco-allemandes, en particulier sur les dynamiques transnationales quotidiennes liant le sport, la politique, la linguistique et l'économie. Auteur des plusieurs ouvrages traitant du dénigrement de l'autre par la langue, de la résilience sociolinguistique et du foot comme vecteur d'une Européanisation.

Marie-Anne Berron - maître de conférences à l'Université de Trèves (Allemagne), responsable de l'enseignement du FLE. Elle a effectué une thèse de doctorat en cotutelle entre l'Université de Paris (Sorbonne) et l'Université de Leipzig. Ses recherches portent non seulement sur la sociolinguistique dans des domaines littéraires, politiques et sportifs par une approche comparée entre le français et l'allemand, mais également sur l'enseignement du français et de l'allemand comme langues étrangères en milieu universitaire et scolaire. 